\title{
SU ALCUNI MATEMATICI NEL SECOLO DEI LUMI IN LOMBARDIA
}

\author{
Nota del m.e. GIANNANTONIO SACCHI LANDRIANI (*)
}

(Adunanza del 3 febbraio 2011)

\begin{abstract}
SunTO. - La tensione culturale che ha caratterizzato il XVIII Secolo è richiamata in questa nota per sottolineare l'opera ed il carattere di alcuni protagonisti, che svolsero la loro attività in Milano dedicandosi essenzialmente a discipline matematiche. Il corsivo allude alla circostanza che il loro campo d'azione spaziò, secondo un costume proprio dei sapienti scientifici di quel tempo, dalla matematica alla fisica, a problemi di ingegneria, alla statica delle costruzioni, alle opere idrauliche, alla meccanica celeste. Milano era, come noto, sede di grandi conquiste sociologiche, giuridiche, economiche e politiche, ad opera soprattutto dei fratelli Pietro ed Alessandro Verri e di Cesare Beccaria, che diedero vita all'Accademia dei Pugni, alla rivista Il caffè, e animarono la corrispondenza con gli Illuministi di massima fama quali d'Alembert, Voltaire, Diderot. Anche l'architettura fu segnata da ben note ancor oggi opere insigni, come ad esempio il Teatro alla Scala del Piermarini. E non si possono dimenticare lo scultore Antonio Canova (statua di Napoleone in Brera), e il grande fisico Alessandro Volta, che chiudono il secolo. Alcuni matematici protagonisti in Lombardia del Secolo dei Lumi, meritano di essere ricordati per l'operosità degna d'ogni riguardo, ma non segnata da grande fama. La citazione della Lombardia è doverosa perché sostegno all'attività scientifica è da attribuirsi all'Università di Pavia, ateneo sede dei personaggi ricordati in questa sede. Si tratta della filantropa cattolica Maria Gaetana Agnesi (Milano 1713-1799), del Gesuita Ruggero Boscovich (Ragusa 1711-Milano 1787), del Barnabita Paolo Frisi (Melegnano 1728-Milano 1784), dell'Abate Lorenzo Mascheroni (Bergamo 1750- Parigi 1800). In questa nota si vogliono tracciare alcuni brevi cenni biografici, peraltro già in parte noti, di questi studiosi. S'intendono altresì sottolineare in bibliografia opere ed autori che in qualche misura, anche indiretta, hanno influito sulla loro attività.
\end{abstract}

$* * *$

(*) Politecnico di Milano.

E-mail: giannantonio.sacchi@polimi.it ; giansacchi.landriani@gmail.com 
ABSTRACT. - During the XIX century in Milano took place, expecially thanks to Cesare Beccaria, Pietro and Alessandro Verri, the developement of the so called "civil economy", the crucial discipline of european Enlightenment. In addition, some mathematics, such as M.G. Agnesi, R. Boscovich, P. Frisi and L. Mascheroni, wrote important works spreading the new teories. However their fame among their contemporaries started to die down with the passing of time, also because coevals of J.L. Lagrange, L. Eulero, J. D’Alembert, still considered indisputed masters.

\section{Maria Gaetana Agnesi}

M. Gaetana Agnesi (Fig. 1), primogenita di una facoltosa famiglia, nasce a Milano nel 1713.

Dotata di straordinaria intelligenza, poco più che ventenne parla e scrive correttamente in italiano, tedesco, francese, latino, greco, spagnolo ed ebraico. A partire dal 1737 si dedica a studi di Filosofia e Matematica (Elementi di Euclide, Logica, Metafisica, Fisica generale e sperimentale).

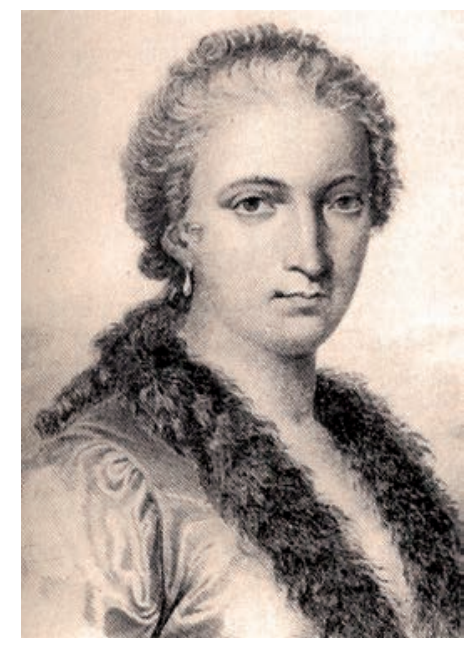

Fig. 1 - M. Gaetana Agnesi.

Nel 1738 pubblica una raccolta di 191 tesi, derivate da pubbliche discussioni, dal titolo Propositiones Philosophicae, collezione enciclopedica in cui sostiene, tra l'altro, la convinzione che anche le donne debbano essere istruite. 
Studia l'opera de l'Hôpital Traité Analytique des Sections Coniques e ne scrive un commento che non risulta pubblicato.

Dal 1740 si dedica allo studio dell'Analisi matematica.

Nel 1748 pubblica, sollecitata da Riccati, ${ }^{1}$ le Instituzioni Analitiche ad uso della Gioventù Italiana (Fig. 2), opera dedicata all'Imperatrice Maria Teresa. Seguirono: una edizione francese nel 1775 ed una edizione inglese nel 1801.

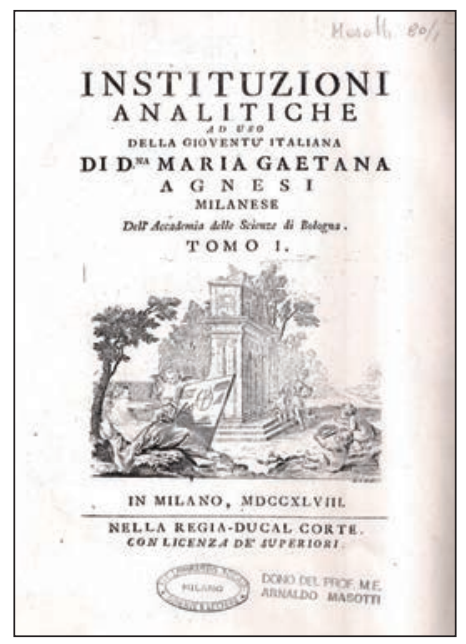

Fig. 2 - Le Instituzioni Analitiche.

Vengono trattate: la teoria delle equazioni algebriche, elementi di geometria analitica piana, calcolo differenziale, regole di integrazione, equazioni differenziali. Nel trattato viene presentata la versiera, curva cubica razionale di equazione cartesiana $y=a^{3} /\left(a^{2}+x^{2}\right)$, simmetrica rispetto all'asse delle $y$, avente come asintoto la retta $\mathrm{y}=0$ e un massimo nel punto $A(0, a)$. L'area compresa tra la curva e l'asse delle $x$ è espressa da $S=\pi a^{2}$ (Fig. 3).

1 Jacopo Riccati (Venezia 1676-Treviso 1754). Si dedicò a problemi di matematica, fisica, idrologia, scienze naturali, storia, filosofia, teologia, pedagogia, architettura, economia. Essenziali i suoi contributi all'analisi matematica soprattutto per l'equazione differenziale $\mathrm{y}^{\prime}=\mathrm{A}(\mathrm{x}) \mathrm{y}^{2}+\mathrm{B}(\mathrm{x}) \mathrm{y}+\mathrm{C}(\mathrm{x})$, all'integrazione della quale si dedicarono Daniele Bernoulli (1700-1782) ed Eulero (1707-1783). 


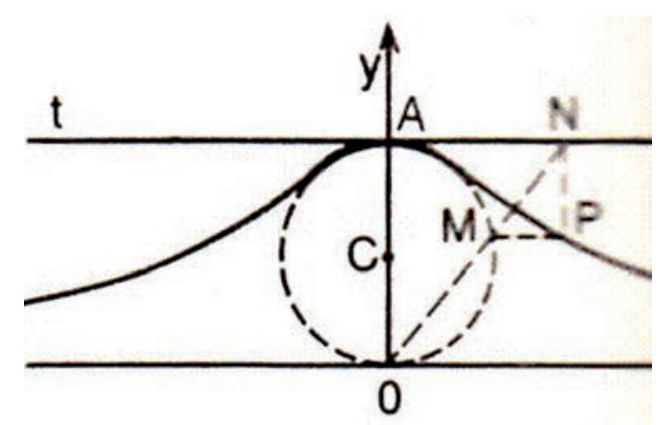

Fig. 3 - La versiera.

La versiera viene ordinariamente attribuita a Gaetana Agnesi, anche se alcuni autori la ritengono scoperta dal matematico camaldolese Guido Grandi (1671-1742). L'attribuzione sembra dovuta alla pubblicazione dell'Agnesi nella sua citata opera.

Gaetana Agnesi rifiutò la cattedra offertale dall'Università di Bologna e a soli 34 anni abbandonò l'attività scientifica per dedicarsi completamente ad opere assistenziali.

\section{Ruggero Giuseppe Boscovich}

Il Gesuita Ruggero Boscovich (Fig. 4) nasce a Ragusa nel 1711 e trascorre la sua vita, ad eccezione di 10 anni passati in Francia, in Italia, dove muore in Milano nel 1787. La sua attività riguardò diverse discipline, forse in armonia con la diffusa cultura enciclopedica. Nel 1740 ebbe l'incarico di insegnamento delle matematiche al Collegio Romano. Affrontò anche problemi di idrografia ed ingegneria. Ne sono testimonianza i suoi studi sulla statica della Cupola di San Pietro, delle guglie del Duomo di Milano, della Biblioteca di Vienna. Esercitò funzioni di diplomatico papale in Europa dal 1750 al 1753 e Benedetto XIV gli affidò il rifacimento delle Carte dello Stato Pontificio.

Nel 1759 si reca in Francia ed in Inghilterra dove viene nominato membro della Royal Society. Nel 1763 compie un viaggio a Costantinopoli per osservare il passaggio di Venere di fronte al Sole. Pubblica successivamente un trattato di Filosofia Naturale. Nello stesso anno 1763 viene nominato professore di Matematiche a Pavia e nel 1764 è invitato a tracciare i piani del nuovo Osservatorio di Brera del 
quale curerà la realizzazione. Successivamente assume la direzione dell'Istituto d'Ottica della Marina francese. Nel 1785 pubblica L'optique et l'astronomie (Fig. 5) e nello stesso anno torna a Milano per dedicarsi all'Osservatorio di Brera.

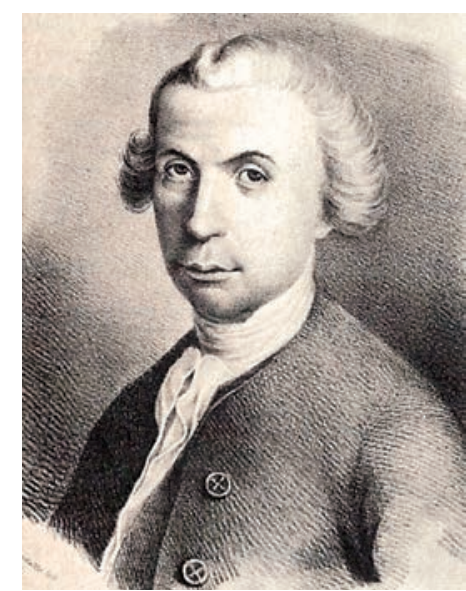

Fig. 4 - Ruggero Boscovich.

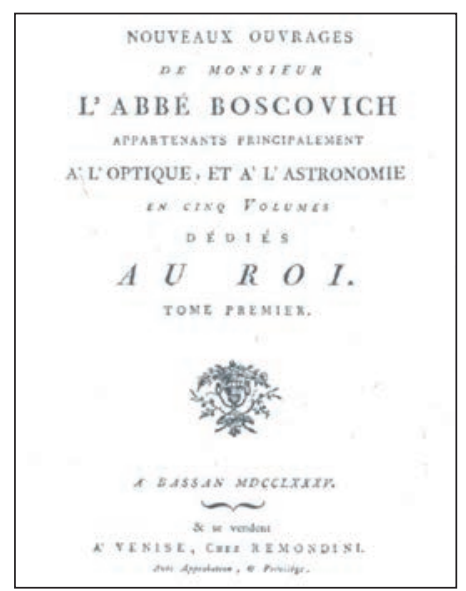

Fig. 5 - Il trattato del 1785.

Nel 1782 fa parte dei fondatori dell'Accademia dei XL.

Gli si può attribuire il merito d'essere stato in Italia uno dei più fermi sostenitori delle teorie gravitazionali di Newton. Si trattò di un vigoroso divulgatore scientifico; tuttavia si espresse con trattazioni, soprattutto di meccanica celeste, di non facile interpretazione e povere di quella chiarezza che il suo impegno avrebbe probabilmente preteso.

Moltissime (77 sino al 2011) le edizioni nazionali di opere di Boscovich, il che testimonia quanto sia stata preziosa la sua eredità culturale essenzialmente nell'astronomia e nella meccanica del sistema solare.

\section{PAOLO FRISI}

La figura del barnabita Paolo Frisi (Fig. 6) è stata decisamente apprezzata in ambito internazionale pur avendo a suo carico una produzione scientifica che definiremmo erudita, ma non dotata di particolare originalità. Ha coltivato molteplici argomenti quali la filosofia 
matematica, l'idraulica, la meccanica celeste, la geodesia, l'algebra, la geometria analitica, il calcolo differenziale. Probabilmente l'eccessiva ampiezza di orizzonti ha caratterizzato la sua notorietà, la sua fama di grande divulgatore soprattutto di Newton e di Eulero. Nel 1764, a soli 36 anni è stato nominato professore delle Scuole Palatine, collaboratore del CAFFE, consigliere del primo ministro dell'imperatrice Maria Teresa, Kaunitz. Nel 1766 compie un viaggio in Europa e soggiorna a lungo in Parigi dove stringe rapporti con scienziati e filosofi. Con D'Alembert terrà, negli anni a seguire, un ricco carteggio. Nel 1773 è socio dell'Académie des Sciences di Parigi, dopo essere stato accolto nel 1757 dall'Accademia Imperiale di Pietroburgo e dalla Royal Society di Londra.

Nel 1758 risulta essere stato chiamato dalle accademie delle scienze di Berlino, di Copenaghen, di Berna, e di aver goduto di una pensione ad honorem dall'Imperatrice d'Austria.

$\mathrm{Fu}$ consultato in tema di gestione di canali ed altri corsi d'acqua, e fu incaricato di redigere il tracciato del Naviglio Milano-Pavia. Pubblicò nel 1781 gli Opuscoli filosofici, trattato il cui sommario (Fig. 7) esibisce compiacimento di singolare ampiezza culturale.

La notevole fama in vita non gli valse grande memoria dei posteri.

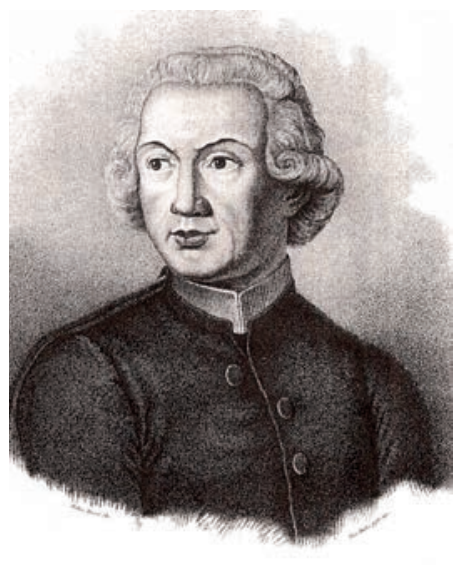

Fig. 6 - Paolo Frisi.

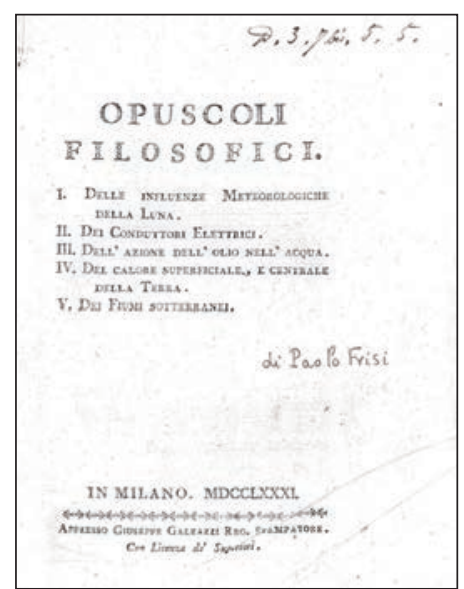

Fig. 7. 


\section{LORENZO MASCHERONI}

Lorenzo Mascheroni (Fig. 8) inizia la sua attività come professore di eloquenza e successivamente di filosofia al Collegio Mariano di Bergamo. Dal 1786 al 1797 ricopre la cattedra di Algebra e Geometria all'Università di Pavia.

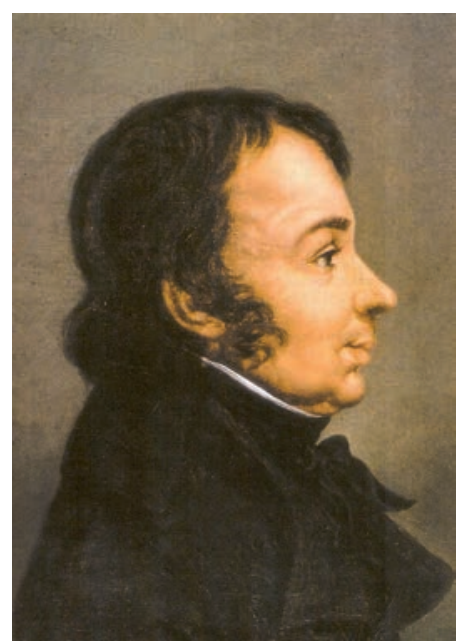

Fig. 8 - Lorenzo Mascheroni.

Mascheroni contribuisce validamente all'interpretazione matematica dell'Ingegneria Civile, ed in particolare della statica delle strutture. Ne è testimonianza il trattato Nuove Ricerche sull'equilibrio delle volte. Attento studioso dell'opera di Eulero pubblica nel 1790 (allora cattedratico all'Università di Pavia) Annotationes ad calculum integralem Euleri. È probabilmente di questo periodo la definizione della cosiddetta Costante di Mascheroni:

$$
\gamma=\lim _{n \rightarrow \infty}\left(\sum_{k=1}^{n} \frac{1}{k}-\ln n\right) \cong 0,57721
$$

Costante che era stata definita anche da Eulero, per cui molti autori preferiscono citarla come costante di Eulero-Mascheroni. Si tratta di un valore notevole quale $\pi$ ed $e$ base dei logaritmi naturali. Significativo risultato della teoria dei numeri e valido strumento in problemi di fisica matematica. 
Nel 1797 pubblica La geometria del compasso, opera nella quale mostra come operazioni di geometria possano essere compiute valendosi soltanto del compasso.

Fervente Bonapartista è membro nel 1797 del Governo della Repubblica Cisalpina. Nel 1798 fa parte della commissione Internazionale dei Pesi e delle Misure.

Oltre che matematico, del quale sono mostrati in figura i frontespizi dei già citati trattati (Figg. 9, 10,11) è anche poeta e di lui si ricorda in particolare Invito a Lesbia Cidonia del 1793, in cui si illustrano in versi, peraltro non fortunati quanto le opere fisico matematiche, i gabinetti scientifici dell'Università di Pavia.

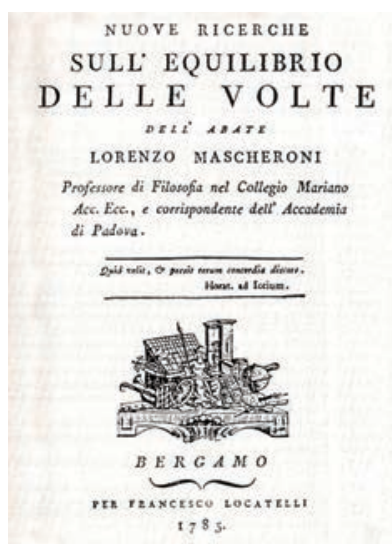

Fig. 9.

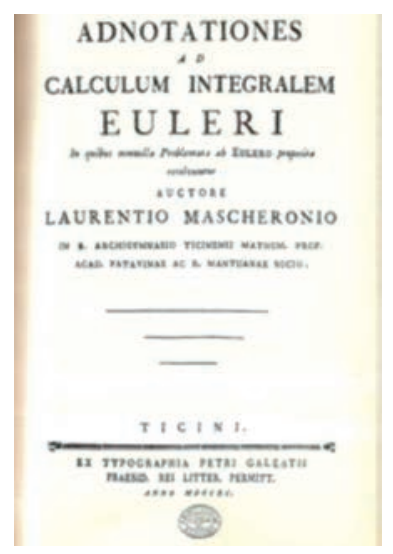

Fig. 10.

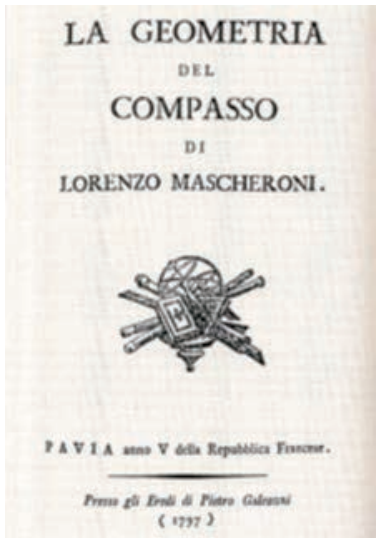

Fig. 11. 


\section{CONSIDERAZIONi E CONGETTURE}

Maria Gaetana Agnesi, Ruggeri Boscovich, Paolo Frisi, Lorenzo Mascheroni furono insigni cittadini e degni uomini di cultura. Alcuni interrogativi sorgono inquadrando le loro personalità nel clima culturale del loro tempo.

Un primo quesito che si offre alla nostra attenzione consiste nella circostanza che si è trattato di quattro religiosi cattolici.

Di Gaetana Agnesi di può dire che fu una sorta di suora laica dedita esclusivamente, dopo i suoi trent'anni, ad opere caritatevoli, in particolare nell'Istituto assistenziale Trivulzi, alla fondazione del quale collaborò e nel quale prese dimora sino al termine della sua vita. Durante il periodo giovanile, di intensa attività matematica, fu vista molto benevolmente dal Vescovo di Bologna, Cardinal Lambertini (assunto al Pontificato col nome di Benedetto XIV), che le propose la cattedra alla Felsinea Università. Cattedra da lei rifiutata.

Di Ruggero Boscovich si può ricordare che fu Gesuita, che si dedicò soprattutto a problemi di astronomia un centinaio d'anni dopo la triste vicenda galileiana e fu attento studioso dell'opera di Newton. Ricevette incarichi in tema di geografia e cartografia nonché di carattere diplomatico dal Papa.

Paolo Frisi fu Barnabita di Sant'Alessandro in Milano ed ebbe corrispondenza fittissima con D'Alembert, Diderot, Voltaire ed in generale con gli ambienti Illuministi del tempo. Visse episodi difficili nei rapporti con il proprio Ordine, non però in tema d'impostazione culturale ma semmai d'osservanza delle regole della Comunità. Ricevette in vita, come abbiamo visto innumerevoli titoli d'onore ed accoglienza da parte di prestigiose Accademie Scientifiche europee.

Quanto a Lorenzo Mascheroni, abate, possiamo osservare che la sua attività di politico e di diplomatico sotto l'egida di Napoleone fu tanto intensa da uguagliare quella di matematico.

Pur essendo vicini come impostazione culturale, e la passione per le scienze esatte lo dimostra, Agnesi, Boscovich, Frisi e Mascheroni non incapparono in richiami delle autorità ecclesiastiche. Il che ci spinge a formulare due semplici congetture.

La prima è che gli ordini ai quali appartenevano erano per antica tradizione depositari di patrimoni culturali ingenti e quindi la possibilità di lavorare e studiare in condizioni di tranquilla riservatezza era assicurata, con la benevola comprensione dei superiori. Non va dimen- 
ticato che grande protettore delle scienze fu, come già è stato osservato, Papa Benedetto XIV.

La seconda è che personalità cattoliche, attirate da un'intensa attività culturale, avevano forse trovato nella matematica una disciplina al riparo da eventuali accuse di eresia. Osservazione tuttavia, per quanto riguarda Boscovich, non del tutto calzante, tenuto conto della di lui intensa attività quale astronomo. Disciplina sulla quale non era del tutto svanita l'ombra del Cardinale Bellarmino.

Una ulteriore osservazione può alludere alla circostanza che i loro contemporanei li avevano considerati degni di grande prestigio. Tuttavia la loro fama di matematici, si è appannata. I matematici moderni citano talvolta la Versiera di Gaetana Agnesi e la Costante di Mascheroni, la cui originalità deve però essere condivisa rispettivamente con Grandi e con Eulero.

Paolo Frisi è ricordato soprattutto per quanto riguarda la meccanica dei fluidi ed in particolare l'idraulica. L'ampio carteggio con D'Alembert costituisce un insieme di documenti il cui interesse è sostanzialmente prezioso nell'ambiente degli studiosi di storia dell'ingegneria e della meccanica applicata.

Un'ultima considerazione riguarda la presenza durante il XVIII secolo di veri giganti della matematica e della fisica matematica, come Eulero, ${ }^{2}$ Lagrange, ${ }^{3}$ D'Alembert ${ }^{4}$ che lasciarono una traccia tanto

2 Leonhard Eulero (Basilea 1701-Pietroburgo 1783). Allievo di Jean I Bernoulli, nel 1727 è membro dell'Accademia di Pietroburgo, nel 1744 direttore della classe di matematica all'Accademia di Prussia, nel 1766 all'Accademia Russa di Pietroburgo. Cultore di Aritmetica ed Algebra, dal 1736 si dedica ad analisi infinitesimale, geometria, meccanica analitica, astronomia. Successivamente ('36-'55) indaga sulla distinzione tra derivate ordinarie e derivate parziali, sull'integrazione delle equazioni differenziali a coefficienti costanti, e sui fondamenti del calcolo delle variazioni. Nel 1770 ottiene un premio sulla teoria dei moti della luna dall'Académie des Sciences di Parigi.

3 Giuseppe Luigi Lagrange (Torino 1736-Parigi 1813). Dal 1756 è professore presso la Regia Accademia d' Artiglieria e Genio di Torino, nel 1758 figura tra i fondatori della Privata Società Scientifica, l'attuale Accademia delle Scienze, di Torino. Nel 1766, proposto da Eulero su sollecitazione di D'Alembert, è chiamato a succedere ad Eulero alla Accademia Prussiana, nel 1787 è chiamato all'Académie des Sciences di Parigi. Nel 1793 è nominato presidente della Commissione del Sistema metrico decimale. E nell'anno successivo collabora alla fondazione dell'Ecole Polytechnique, dove (nel '97) è il primo professore di analisi matematica. E' nominato Conte da Napoleone 
profonda da essere ancora oggi degna d'indagine scientifica. Studiosi rigorosi e critici di grandi scienziati (quali Galileo (1564-1642), Newton (1642-1727), Leibnitz (1646-1716), Daniele Bernoulli (1700-1782) aggiunsero contributi originali a quelli lasciati dai loro illustri predecessori.

Lasciarono un'ombra tanto estesa da oscurare i pregi di eruditi che pur operarono con intenso ed onesto impegno. ${ }^{5}$

Bonaparte ed è sepolto al Panthéon di Parigi. Si era dedicato alla definizione del calcolo delle variazioni, alla teoria delle funzioni, alla meccanica celeste. Opera fondamentale può ritenersi Mécanique Analytique, la cui prima edizione è del 1788, contenente espressioni fondamentali dal Principio dei Lavori Virtuali, alla definizione dei moltiplicatori di Lagrange, ai capitoli su les libraisons de la lune e alla stabilità del sistema solare.

4 Jean-Baptiste D'Alembert Le Rond (Parigi 1717-1783). Nel 1730 è eletto membro dell'Académie des Sciences di Parigi, e nel 1772 ne diviene Secrétaire Perpétuel. Memorabili i suoi studi sulle Corde vibranti, sulla dinamica dei solidi, sulla formulazione (1773) del cosiddetto Principio di D'Alembert, che consente di interpretare un sistema dinamico con metodi propri della statica. A partire dal 1749 si dedica a problemi di meccanica celeste ed in particolare alla precessione degli equinozi. Sono intensi i carteggi con Lagrange, Eulero, Frisi, Voltaire, Rousseau, Beccaria, Federico II. Il suo alto prestigio deriva anche dalla pubblicazione, con Diderot, a partire dal 1751 de l'Encyclopedie recante il suo Discours Préliminaire des éditeures.

5 È opportuno ricordare alcuni dei grandi magnati della scienza matematica dell'epoca: Papa Benedetto XIV (1657-1758), Maria Teresa d'Austria (1717-1780), Giuseppe II d'Austria (1741-1790), Federico II il Grande di Prussia (1721-1786), Caterina II di Russia (1729-1796), Luigi XVI di Francia (1754-1793), Napoleone I Bonaparte (1769-1821). 


\section{RIFERIMENTI BIBLIOGRAFICI}

M.G. Agnesi, Propositiones Philosophicae, quas crebris disputationibus domi habitis coram clarissimis viris explicabat extempore, et ab objectis vindicabat. Milano, 1738 .

M.G. Agnesi, Istituzioni analitiche ad uso della gioventù italiana, voll. 2, Milano, 1748 IL.

M.G. Agnesi, Analytical Institutions in four books..., Vol. I, Engl.Transl.

F. Algarotti, Opere Scelte, Volume Secondo contente: Dialoghi Sopra l'Ottica Neutoniana, Poesie Epistole, Odi. Potzdam 1752. Ed. Milano, 1823 IL.

R.J. Boscoivch, De motu corporis attracti in centrum immobile viribus, decrescentibus in ratione distantiarum reciproca duplicata in spatii non resistentibus. Roma, Typis Komarek, 1743. IL.

R.J. Boscovich, De inequalitatibus quas Saturnus et Jupiter sibi mutuo videntur inducere presertim circa tempus conjunctionis. Roma, 1756 IL.

R.J. Boscovich, Elementorum universae matheseos Tomus I continens: Geometriam Planam, Aritmeticam Vulgarem, Geometriam Solidarum, Trigonometriam Planam, E Sphericam, Venetiis, 1757 IL.

R.J. Boscovich, Tomus II continens: Algebram Finitam, Venetiis, 1757 IL.

R.J. Boscovich, Tomus III continens: Sectionum Conicarum Elementa nova quadam methodo concinnata \& Dissertatitionem de Transformatione Locorum Geometricorum, ubi de Continuitatis lege, ac de quibusdam Infiniti Mysteriis. Venetiis, 1757 IL.

R.J. Boscovich, Del porto di Rimini, Raccolta da Autori Italiani... del moto dell'acque, tomo VII, Bologna, 1823 IL.

R.J. Boscovich, De solis ac lunae defectibus. Libri V. Venetiis, 1761 IL.

R.J. Boscovich, Theoria philosophiae naturalis redacta ad unicam legem virum in natura existentium, Venezia, 1763 IL.

R.J. Boscovich, Opera pertinentia ad opticam et astronomicam maxima ex parte nova et omnia buiusque inedita. Bassani, 1785 IL.

M. Bossi e P. Tucci Eds, Bicentennial commemoration of R.G. Boscovich, Milano, 1988 IL.

J. D’Alembert Le Rond, Traité de l'equilibre et du movement des fluids. Pour server de suite a Traité de dynamique. Paris, 1744 IL.

J. D'Alembert Le Rond, Recherches sur la précession des équinoxes et sur la mutation de l'axe de la terre dans le système newtonien, Paris, 1749 IL.

J. D’Alembert Le Rond et D.J. Diderot, Encycolpedie ou Dictionnaire Raisonné des Sciences, Des Arts et Métiers, Paris, 1751 IL.

J. D'Alembert Le Rond, Recherches sur différens points importants du système du monde, 3 voll. Paris, 1754-1756 IL. 
J. D’Alembert Le Rond, Recherches sur different points importants du système du mond, Paris, 1754 IL.

J. D’Alembert Le Rond, Traité de Dynamique, Paris, 1758 IL.

J. D'Alembert Le Rond, Histoire des membres de l'Académie françoise morts depuis 1700 jusqu'au 1771, Amsterdam, 1787, 5 voll. di 6, IL.

L. Eulero, Mechanica sive motus scientia analytice exposita, Petropoli, Acad. Scientiarum, 2 voll., 1736 IL.

L. Eulero, Lettres a une princesse d'Alemagne sur divers sujets de physique et de philosophie... avec une introduction et des notes par Emil Saisset. Paris, 1843 IL.

L. Eulero, Methodus inveniendi lineas curvas maxima minive proprietate gaudentes, sive solutio problematis isoperimerimetrici latissimo sensu accepti, Lausanne et Genevae, 1744 IL.

L. Eulero, Eléments d'algèbre, 2 voll., Lyon, 1774 IL.

L. Eulero, Introductio in anlysis infinitorum, 2 voll. Lausanne, 1748 IL.

L. Eulero, Theoria motus corporum solidorum seu rigidorum, Rostochii, 1765 IL.

L. Eulero, Institutionum calculi integralis, 4 voll., Petropoli, 1768-1794 IL.

L. Eulero, Saggio di una difesa della divina rivelazione; traduzione de tedesco coll'aggiunta dell'esame dell'argomento dedotto dall'abbreviamento dell'anno solare e planetario, Pavia, 1777 IL.

L. Eulero, Institutiones calculi differentialis cum eius usu in analysis finitorum ac doctrina serierum, Ticini, 1787 IL.

P. Frisi, Elogio di Bonaventura Cavalieri, Donato Silva, Galileo Galilei, Isacco Newton, Milano, 1829 IL.

P. Frisi, Dei fiumi, dei torrenti, dei canali navigabili, Raccolta da Autori Italiani... del moto dell'acque, tomo VI, Bologna, 1823 IL.

P. Frisi, Opuscoli filosofici: I. Dell'influenze metereologiche della Luna; II. Dei conduttori elettrici; III. Dell'azione dell'olio nell'acqua; IV. Del caloer superficiale e centrale della Terra; $V$. Dei fiumi sotterranei, Milano, 1781IL.

P. Frisi, Elogio di Maria Teresa Imperatrice, I quaderni di Palazzo Sormani n.4, Milano, 1981.

A.F. Frisi, Elogio storico di D. ${ }^{A}$ Maria Gaetana Agnesi, Milano 1799, ristampa critica a cura di Arnaldo e Giuseppina Masotti, Milano, 1965 IL.

Galluzzi M., Paolo Frisi, d'Alembert et le milieu scientifique de Milan, Storia delle Scienze matematiche, $\mathrm{n}^{\circ}$ 2, Pisa, 2008.

A.Gigli Berzolari Lorenzo Mascheroni, Milano, 2001 IL.

J.L. Lagrange, Méchanique Analitique, $1^{\text {ere }}$ Ed. Paris, 1788 IL.

J.L. Lagrange, Théorie des fonctions analytiques, contenant les principes du calcul differentiel, dégagés de toute conmsidération d'infintement petits ou évanouissans, de limites ou de fluxions et réduits a l'analyse algébrique des quantités finies, Priarial an V IL.

J.L. Lagrange, Mécanique Analytique, $2^{\mathrm{eme}}$ Ed. 2 vol. Paris, 1815 IL. 
L. Mascheroni, Adnotationes ad calculum integralem Euleri, in duos partes divisos. Ticini, 1790-92. IL.

L. Mascheroni, La geometria del compasso, Pavia, 1797. IL.

L. Mascheroni, Problemi di Geometria colle dimostrazioni aggiunte dal cittadino Sacchi, Milano, 1802. IL.

L. Mascheroni, Problemi di Geometria. Milano, anno X. Legato con: 1) Puissant. L.: Recueil de propositions de géometrie e con Johns Colson, London, 1801 IL; 2)Boucharlat J.L.: Remarques sur l'algebre. IL.

L. Mascheroni, Invito a Lesbia Cidonia ed altre poesie, Milano, 1887; Torino, 1915. IL.

A. Masotti, Matematica e matematici nella storia di Milano, Rend. Seminario matematico e Fisico di Milano, Vol.XXXIII, Pavia, 1963.

A. Masotti, Maria Gaetana Agnesi, Rendiconti del seminario matematico e fisico, Vol. 14, Milano, 1940 IL.

L. Pepe, Lorenzo Mascheroni, memorie analitiche, Bergamo, 2000 IL.

L. Pepe, Istituti nazionali, Accademie e Società scientifiche nell'Europa di Napoleone, Firenze, 2005.

L. Pepe, Rinascita di una scienza. Matematica e matematici in Italia (1715-1814), Bologna, 2007.

A. Piccato, Dizionario dei termini matematici, Milano, 1987.

E. Proverbio E. (a cura di) Acc.Sc.XL, INAF, Osservatorio Brera, Pontif. Univ. Gregoriana, Ed. Naz. Opere di Boscovich, 2006 IL.

Le voci recanti la sigla IL sono relative ad opere custodite nella biblioteca della nostra Accademia di Scienze e Lettere.

The works marked IL are collected at the Istituto Lombardo library. 